\title{
Processus De Creation D’une Zone De Conservation De La Biodiversite Sur Le Barrage Hydroelectrique De Soubre, Sud- Ouest De La Côte D’ivoire
}

\author{
Yao Jean-Clovis Kouadio, Assistant \\ Abdoulaye Cissé, Assistant \\ Université Félix Houphouët-Boigny, Côte d’Ivoire \\ Kouassi Bruno Kpangui, Maître Assistant \\ Université Jean Lorougnon Guédé, Côte d’Ivoire \\ Marie-Solange Tiébré, Professeur Titulaire \\ Djakalia Ouattara, Professeur Titulaire \\ Centre National de Floristique, Côte d'Ivoire \\ Kouakou Edouard N'Guessan, Professeur Titulaire \\ Université Félix Houphouët-Boigny, Côte d’Ivoire
}

\section{Doi:10.19044/esj.2021.v17n40p56}

Submitted: 26 June 2021

Accepted: 01 October 2021

Published: 30 November 2021
Copyright 2021 Author(s)

Under Creative Commons BY-NC-ND 4.0 OPEN ACCESS

Cite As:

Kouadio Y.JC.,Cisse A., Kpangui B. K., Tiebre M., Ouattara D.,\& N’Guessan E. K., (2021). Processus De Creation D'une Zone De Conservation De La Biodiversite Sur Le Barrage Hydroelectrique De Soubre, Sud-Ouest De La Côte D'ivoire. European Scientific Journal, ESJ, 17(40), 56.

https://doi.org/10.19044/esj.2021.v17n40p56

\section{Résumé}

Cette étude a pour objectif de montrer l'importance de la participation des communautés locales à la réussite d'un projet de création de zone de conservation de la biodiversité. La mise en place de ladite zone a nécessité la formation des autorités et communautés locales sur les services écosystémiques. Cette formation a permis une meilleure implication des communautés à la gestion durable des ressources. Ainsi, 20 représentants ont servi de personnel d'appui pour les travaux d'aménagement et de restauration du site. L'état initial de la flore et la végétation a été évalué. L’analyse des données a mis en évidence la présence de 346 espèces végétales reparties en 262 genres et 89 familles. Parmi celles-ci il a été enregistré 50 espèces prioritaires pour la conservation. Au sein des espèces utilitaires de la région, 13 sont plus importantes. Cependant, six d'entre elles sont moins abondantes 
dans la zone de conservation de biodiversité. La caractérisation de la flore initiale a guidé dans le choix des espèces de reboisement pour la restauration. Ainsi, les espèces prioritaires pour la conservation et celles désignées importantes pour la population ont été privilégiées au cours de la restauration. Les résultats obtenus renseignent d'une part sur les outils d'acceptation sociale et les niveaux d'implication de la communauté locale d'un projet de mise en place d'une zone de conservation de biodiversité et d'autre part sur l’importance de la caractérisation de la flore initiale.

Mots clés: Biodiversité, Conservation, Restauration, Soubré, Côte d’Ivoire

\title{
Process For the Creation of a Biodiversity Conservation Area on the Soubre Hydroelectric Dam, Southwestern Ivory Coast
}

\author{
Yao Jean-Clovis Kouadio, Assistant \\ Abdoulaye Cissé, Assistant \\ Université Félix Houphouët-Boigny, Côte d’Ivoire \\ Kouassi Bruno Kpangui, Maître Assistant \\ Université Jean Lorougnon Guédé, Côte d'Ivoire \\ Marie-Solange Tiébré, Professeur Titulaire \\ Djakalia Ouattara, Professeur Titulaire \\ Centre National de Floristique, Côte d'Ivoire \\ Kouakou Edouard N'Guessan, Professeur Titulaire \\ Université Félix Houphouët-Boigny, Côte d’Ivoire
}

\section{Abstract}

This paper focuses on showing the importance of the participation of local communities in the success of a project to create a biodiversity conservation area. The establishment of this zone required the training of local authorities and communities on ecosystem services. This training allowed for a better involvement of the communities in the sustainable management of resources. Twenty (20) community representatives served as support staff for the development and restoration of the site. The initial state of the flora and vegetation was assessed. The analysis of the data revealed the presence of 346 plant species divided into 262 genera and 89 families. Of these, 50 priority species for conservation were recorded. Of the utilitarian species of the region, 13 are more important. Six of them are less abundant in the biodiversity conservation area. The characterisation of the initial flora guided the selection of reforestation species for restoration. Priority species for conservation and those designated as important for the population were favoured during the restoration. The results obtained provide information, on the one hand, on the 
tools for social acceptance and the levels of involvement of the local community of a project to set up a biodiversity conservation area and, on the other hand, on the importance of characterizing the initial flora.

Keywords: Biodiversity, Conservation, Restauration, Soubré, Côte d'Ivoire

\section{Introduction}

La couverture forestière de la Côte d'Ivoire s'est considérablement dégradée au cours des dernières années. De 16 millions d'hectares de forêts au début du $20^{\text {ème }}$ siècle (Lanly, 1969), les superficies résiduelles de forêts ne représentent plus qu'environ 3,4 millions d'hectares en 2015 (FAO-REDD+, 2016).

L'agriculture, principale base du développement économique de la Côte d'Ivoire, qui reste extensive et consommatrice de terres forestières est l'une des causes directes de la perte de la couverture forestière (Konan, 2008). Parallèlement aux causes directes, il existe d'autres facteurs qui impactent négativement le couvert forestier national. On peut noter le développement des infrastructures (routes, barrages hydroélectriques...) et le développement minier avec la pratique de l'orpaillage clandestin qui détruit les forêts et pollue les cours d'eau (MINEF, 2020).

Avec la croissante démographique et l'immigration des populations de la sous-région vers les terres forestières, les pressions sur les forêts seront de plus en plus fortes si l'Etat ne s'engage pas fortement dans la sauvegarde de la forêt. Ainsi, pour inverser cette tendance à la dégradation, aux assemblées des nations unies sur le changement climatique en 2014 à New York, l'Etat de Côte d'Ivoire s'est engagé dans une transition vers une agriculture zéro déforestation à partir de 2017. Par la suite, il a adopté une politique de préservation, de réhabilitation et d'extension des forêts le 23 mai 2018 (Communiqué du conseil des ministres du 23 mai 2018). Pour mettre en œuvre cette politique, une stratégie nationale a été élaborée. L’objectif national de cette stratégie revient en la reconstitution du couvert forestier d'au moins $20 \%$ du territoire national à l'horizon 2045, tout en améliorant les conditions de vie des populations par la création d'emplois verts et la restauration des sols (MINEF, 2020). Pour atteindre cet objectif, l’Etat a engagé différents acteurs à fournir davantage d'efforts pour la sauvegarde des forêts. Ainsi, il a encouragé d'une part les agriculteurs à la pratique de l'agroforesterie et d'autre part, les communautés à la création de réserves naturelles volontaires et les entreprises au développement de stratégies et politiques vis-à-vis de la biodiversité. Ainsi, les entreprises devraient prendre en compte l'environnement dans la planification ou le développement des projets en vue d'assurer la protection de l'environnement et la conservation des milieux de vie. C’est dans ce cadre que pendant la mise en œuvre du projet de construction du barrage hydroélectrique de Soubré sur le fleuve Sassandra par le promoteur 
CI-ENERGIE, un espace de 200 ha a été dédié à la création d'une zone de conservation de la biodiversité. Les aménagements nécessaires et la restauration qui en ont suivi ont permis de disposer d'une zone de conservation de la biodiversité. Malheureusement le processus de mise en place reste peu documenté et mal connu. La question principale est de savoir comment convaincre les populations à céder une partie de leurs terres face à une demande accrue de terres cultivables. De cette question découlent les questions de recherche subsidiaires. Quels ont été les outils d'acceptation sociale du projet? Quels sont les aménagements nécessaires à la création d'une zone de biodiversité ?

La présente étude tente d'apporter des éléments de réponse à ces différentes questions. Son objectif général est de montrer l'importance de l'implication des communautés locales à la réussite d'un projet de création de zone de biodiversité. De façon spécifique il s'agit d'une part d'identifier les outils d'acceptation sociale du projet et d'autre part de situer les niveaux d'implication des communautés locales.

\section{Matériel et méthodes}

La zone de conservation de la biodiversité est située dans la région de la Nawa. Le chef-lieu de région est la ville de Soubré. Elle a été mise en place dans le cadre de l'implémentation du Plan de Gestion Environnemental et Social (PGES) du projet de construction du barrage hydroélectrique de Soubré afin de compenser les impacts sur la perte de la biodiversité. Elle est précisément en aval immédiat du barrage hydroélectrique et constitue un continuum écologique de 200 ha reparti sur les rives gauche et droite du fleuve Sassandra (Figure 1).

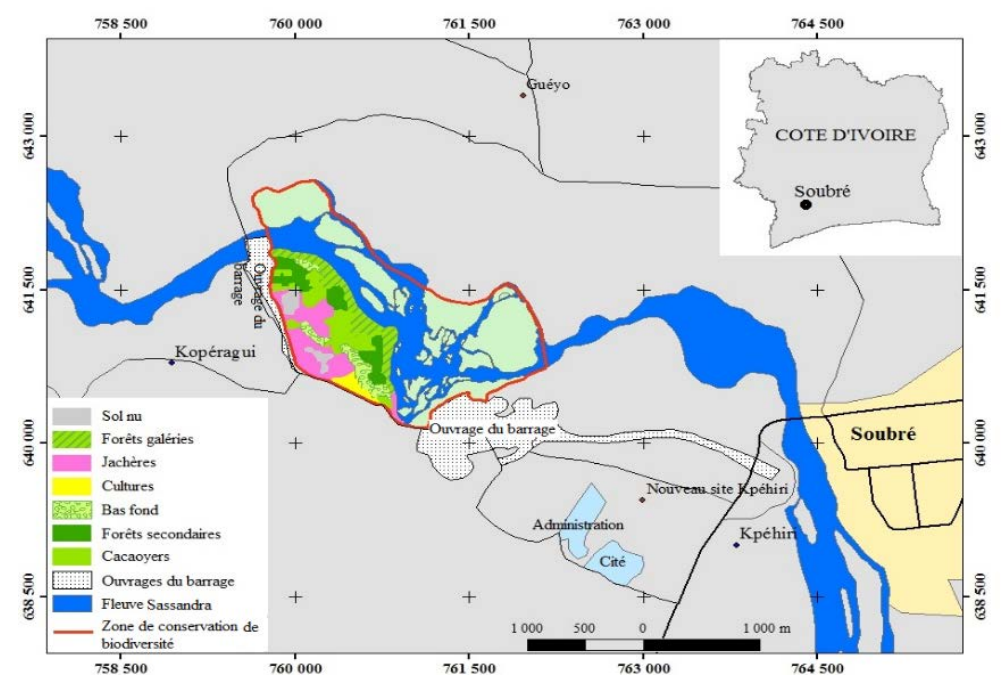

Figure 1. Présentation du site de conservation de la biodiversité 
Au cours du processus de création de ladite zone, plusieurs activités ont été menées. Ces activités ont débuté par la formation des autorités administratives locales et les populations sur les biens et services rendus par les écosystèmes. Cette formation s'est achevée par le recrutement d'un personnel d'appui au nombre de 20 personnes issues des différents villages. Ces derniers ont suivi des séances de formation aux processus de création d'une zone refuge de biodiversité et d'une pépinière.

Quant aux activités d'aménagement du site, avec l'aide du personnel d'appui, elles ont débuté par la réalisation du layon de délimitation et du sentier botanique suivant différentes curiosités botaniques. Par la suite, une collecte de semences a été entreprise dans le réservoir du barrage bien avant la mise en eau. Les semences collectées (graines et plantules) ont servi à la réalisation d’une pépinière.

Afin d'évaluer, à l'état initial, la composition floristique de la végétation de la zone, deux techniques de relevés de terrain ont été utilisées. Il s’agit du relevé de surface et du relevé itinérant. Ces deux types de relevés sont complémentaires. Le relevé de surface a consisté à délimiter des placettes de $20 \mathrm{~m}$ x $20 \mathrm{~m}\left(400 \mathrm{~m}^{2}\right)$ chacune dans les différents types de biotopes rencontrés. A l'intérieur de chaque placette, il a été identifié d'abord le type de végétation. Par la suite, toutes les espèces de plantes rencontrées ont été inventoriées. Les relevés itinérants n’ont concerné que les espèces non rencontrées dans les relevés de surface. Les espèces observées, dans ce deuxième type d'inventaire, ont été notées et des échantillons récoltés pour compléter la liste floristique générale.

Afin de tenir compte de leur avis dans le choix des espèces devant servir au reboisement, une enquête ethnobotanique a été réalisé auprès des populations riveraines de la zone de conservation de biodiversité. Elle a porté sur l'identification des plantes utilitaires de la région. Les personnes ressources identifiées pour mener à bien cette étude étaient composées de praticiens de la médecine traditionnelle et des personnes disposant des connaissances locales sur les plantes.

\section{Méthodologie d'analyse des données}

Richesse et composition floristique : Pour chacune des espèces inventoriées, il a été associé la famille, le genre, le type biologique et la répartition phytogéographique. Toutes les espèces ont été identifiées grâce aux travaux de Aké-Assi (1984) et de Lebrun \& Stork (1991-2015). La nomenclature adoptée pour l'actualisation des noms et familles est celle d'APG IV (2016). Par la suite, les espèces ont été classées en épiphytes (Ep), géophytes (G), hémicryptophytes (H), thérophytes (Th), chaméphytes (Ch) et phanérophytes. Ce dernier groupe comprend les nanophanérophytes (np), arbrisseau de 0,25 à $2 \mathrm{~m}$ de hauteur ; les microphanérophytes (mp), arbuste de 
2 à $8 \mathrm{~m}$ de hauteur ; les mésophanérophytes (mP), arbre de 8 à $30 \mathrm{~m}$ de hauteur ; et les mégaphanérophytes (MP), arbre de plus de $30 \mathrm{~m}$ de hauteur. En ce qui concerne la répartition phytogéographique, il s'agit de préciser comme l'on définit plusieurs auteurs dont Aké-Assi (1984), les espèces qui se rencontrent naturellement dans la région phytogéographique Guinéo-Congolaise (GC) ou dans la région phytogéographique Soudano-Zambézienne (SZ), ou à la fois dans ces deux régions phytogéographiques (GC-SZ) ou encore les espèces introduites (i). Pour l'endémisme, il a été distingué les endémiques aux blocs forestiers Ouest africains (GCW) parmi lesquelles celles qui sont propres au territoire ivoirien sont désignées par GCi (Aké-Assi, 2001 ; Aké-Assi, 2002 ; Poorter et al., 2004). Les espèces endémiques aux forêts de la région phytogéographique de la Haute Guinée sont désignées par HG.

Enfin, la liste floristique obtenue a été croisée, en premier lieu, avec la liste des espèces utilitaires de la région obtenue à partir de l'enquête ethnobotanique afin de déceler leur disponibilité, en second lieu, avec la liste rouge de l'UICN (2018) et celle des espèces rares et menacées de Aké-Assi (1998). Ce travail a permis d'obtenir une liste complète des espèces qui renseigne toutes les informations nécessaires pour chaque espèce. Ainsi, les espèces endémiques GCW, GCi et HG et les espèces à statut de conservation selon UICN (2018) et Aké-Assi (1998) ont été considérées comme prioritaires pour la conservation dans la zone de biodiversité.

Valeur d'importance des espèces: La valeur d'importance (VIsp) de l'espèce représente le rapport entre le nombre d'usages différents pour l'espèce (vi) et le nombre d'usages différents pour l'ensemble des espèces répertoriées $(\Sigma v i)$. Elle se calcule selon la formule suivante:

$V I s p=\frac{v i}{\sum v i} \times 100$

Cette valeur permet de mettre en exergue les espèces sollicitées dans plusieurs domaines et qui auraient de ce fait une importance dans la communauté.

Evaluation de la disponibilité des espèces : Elle a été évaluée à partir de l'indice de rareté des espèces. Cet indice se calcule en utilisant la formule suivante (Géhu \& Géhu, 1980).

$$
R i=1-\left(\frac{n i}{N i}\right) \times 100
$$

Dans cette équation $R i$ représente l'indice de raréfaction d'une espèce $\mathrm{i}$ ; ni le nombre de parcelle où elle est rencontrée et $\mathrm{N}$ le nombre total de parcelles inventoriées.

Les espèces dont les indices de raréfaction sont inférieurs à $80 \%$ sont considérées comme préférentielles, très fréquentes et abondantes dans les zones étudiées. Celles dont les indices de raréfaction sont supérieurs à $80 \%$, sont dites rares et donc fortement menacées d'extinction dans la localité. 


\section{Résultats}

\section{Personnes formées sur les biens et services écosystémiques}

Plusieurs personnes ressources de la région ont été formées. Outre les autorités administratives, les représentants de tous les villages impactés y ont été impliqués. Un total de 105 personnes issues des communautés villageoises et 11 autorités administratives a été enregistré. La formation leur a permis de mieux comprendre l'intérêt de la conservation de la diversité biologique pour leur bien-être et les bienfaits d'une gestion durable des forêts afin qu'ils se convertissent en défenseur de la biodiversité dans leurs villages respectifs.

\section{Aménagements effectués dans la zone de biodiversité}

Un sentier botanique long de 2,24 km permet de pratiquer l'écotourisme au sein de la zone de biodiversité. Sur le parcours l'on rencontre des espèces prioritaires pour la conservation inscrites sur la liste rouge de l’UICN (2018) et la liste nationale Aké-Assi (1998) notamment Copaifera salikounda Heckel., Milicia regia (A. Chev.) C.C. Berg., Nesogordonia papaverifera (A. Chev.) Capuron ex N. Hallé (Annexe 1). Elles sont étiquetées pour faciliter leurs identifications par les visiteurs. Le sentier comporte également deux aires de repos équipées de tabourets qui sont des rondelles de tiges devant servir de banquettes pendant les séances de repos des visiteurs. Aussi, deux rangées de teck (Tectona grandis L. f.) permettent-elles de marquer les limites avec le domaine rural. Les individus sont espacés de 2,5 $\mathrm{m}$ sur les lignes et 5 $\mathrm{m}$ en interligne.

\section{Plants produits pour le reboisement}

Une pépinière composée de plants de teck et d'espèces prioritaires pour la conservation a été mise en place. On dénombre 2.300 plants de teck et 20.000 plants d'espèces à haute valeur d'usage et/ou d'espèces prioritaires pour la conservation selon UICN (2018). On peut citer en autres Mlicia regia (A. Chev.) C.C. Berg (Moraceae), Ricinodendron heudelotii var. Africanum Baill. Pierre ex Heckel (Euphorbiaceae), Xylopia aethiopica (Dunal) A. Rich. (Annonaceae), Tieghemella heckelii (A. Chev.) Pierre ex Dubard (Sapotaceae), Khaya ivorensis A. Chev. (Meliaceae), Blighia sapida K.D. Koenig (Sapindaceae).

\section{Végétation et flore initiale du site}

Types d'habitats rencontrés: Les forêts galeries, les forêts secondaires, les formations marécageuses, les jachères et les espaces anciennement cultivés sont les principaux biotopes rencontrés. Les forêts galeries bordent les différents affluents du fleuve Sassandra. Leur composition floristique est souvent assez particulière. Selon leur niveau de conservation, l'on y rencontre des espèces caractéristiques telles que Uapaca heudelotii Baill., Uapaca guineensis Müll. Arg., Macaranga spp, Napoleonaea vogelii Hook. \& Planch. et Pterocarpus santalinoides L'Hér. ex DC. Les forêts secondaires se rencontrent entre les plantations cacaoyères et en arrière-plan 
des galeries forestières. Des espèces telles que Ceiba pentandra (L.) Gaertn. et Piptadeniastrum africanum (Hook. f.) Brenan se rencontrent dans la strate supérieure. La strate secondaire est représentée par des espèces héliophiles telles que Musanga cecropioides R. Br., Ficus exasperata Vahl, Sterculia tragacantha Lindl. La strate basse compte des herbacées et d'autres espèces comme Chromolaena odorata (L.) R.M. King \& H. Rob., Solanum rugosum Dunal, Trema orientalis (L.) Blume. Les formations marécageuses se rencontrent dans les zones de bas fond sur sols hydromorphes. Certaines de ces formations végétales peuvent être qualifiées de forêt à Raphia compte tenu de l'abondance des individus de cette espèce. Elles présentent un bon niveau de conservation. Les espèces les plus fréquentes de la strate supérieure sont: Raphia hookeri G. Mann \& H. Wendl., Mitragyna ledermannii (K. Krause) Ridsdale et Uapaca guineensis Müll. Arg. La strate inférieure est dominée par Alchornea cordifolia (Schumach. \& Thonn.) Müll. Arg, Palisota hirsuta (Thunb.) K. Schum., Nephrolepis biserrata (Sw.) Schott, et des rotins tels que Laccosperma secundiflorum (P. Beauv.) Kuntze et Calamus deerratus G. Mann \& $\mathrm{H}$. Wendl.. Les jachères constituent la formation végétale la plus abondante. On y rencontre de nombreuses espèces héliophiles. Quelques grands arbres tels que Albizia adianthifolia (Schumach.) W. Wight y sont rencontrés. Enfin, on rencontre des espaces anciennement cultivés. Il s'agit des plantations d'hévéa et de cacaoyers. On y observe une reprise de la végétation marquée par de jeunes pousses d'espèces comme Antiaris toxicaria var. africana Scott-Elliot ex A. Chev. var. africana, Milicia excelsa (Welw.) C.C. Berg, Pycnanthus angolensis (Welw.) Warb., Tetrapleura tetraptera (Schumach. \& Thonn.) Taub. et Albizia adianthifolia (Schumach.) W. Wight, etc.

Richesse et composition floristique : La présente étude a révélé la présence de 346 espèces végétales. Elles se répartissent en 262 genres et 89 familles. Les familles les plus riches en espèces sont celles des Fabaceae (25 espèces), Rubiaceae (22 espèces), Euphorbiaceae (19 espèces), Poaceae (17 espèces), et Moraceae (15 espèces).

Au total, 11 types biologiques ont été recensés sur le site du projet. Ce sont les Megaphanérophytes, Mesophanérophytes, Microphanérophytes, Nanophanérophytes, Chaméphytes, Thérophytes, Hemicryptophytes, Geophytes, Géophytes rhizomateux, Hydrophytes et les Hémicryptophytes pyrophytique. Les espèces Microphanérophytes (mp) sont les plus prépondérantes. Elles sont représentées par 158 espèces, soit 45,40 \% des espèces.

L'ensemble de la zone de conservation est dominé par les espèces qui se rencontrent en région Guinéo-Congolaise (GC). Ces espèces représentent $58,04 \%$ de toutes les espèces recensées. Elles sont suivies des espèces de la zone de transition (GC-SZ). 
Espèces prioritaires pour la conservation : parmi les espèces recensées, 50 ont un statut de conservation (espèces endémique et espèces rares et menacées d'extinction) soit 14,36 \% de la diversité (Annexe 1). On estime à 38 l'ensemble de celles qui sont endémiques (GCW, GCi, HG) dont 5 endémiques à la Côte d'Ivoire (GCi). Ce sont entre autres Baphia pubescens Hook. f., Leptoderris miegei Aké Assi \& Mangenot, Eugenia miegeana Aké Assi. Les espèces endémiques à la zone de Haute-Guinée (HG) sont au nombre de 28. L'on a recensé 27 espèces endémiques appartenant au bloc forestier de l'Afrique de l'Ouest (GCW). A titre d'exemple, on peut citer Rinorea subintegrifolia (P. Beauv.) O. Ktze et Tiliacora dinklagei Engl. Les espèces menacées inscrites sur la liste rouge de l'UICN (2018) sont au nombre de 10. Elles sont toutes considérées comme vulnérables. Ce sont entre autres Albizia ferruginea (Guill. \& Perr.) Benth., Craibia atlantica Dunn. Au plan national, 8 espèces recensées sont considérées comme des espèces rares et en voie d'extinction selon Aké-Assi (1998). Il s'agit entre autres de Garcinia afzelii Engl (Clusiaceae), Maranthes robusta (Oliv.) France (Chrysobalanaceae), Pararistolochia mannii (Hook.f.) Keay (Aristolochiaceae), Milicia excelsa (Welw.) Berg.

\section{Catégories d'usage des espèces utilitaires citées par les populations}

Les espèces utilitaires ont été rangées en plusieurs catégories d'usage. Ce sont, par ordre d'importance, l'usage médicinal (71 espèces), l'usage alimentaire (28 espèces), l'artisanat (20 espèces), le bois d'œuvre qui concerne 14 espèces (Figure 2). Divers autres usages tels que l'ornementation, le fourrage, insecticide, accessoires de pêche et de chasse, emballages sont également cités et concernent 11 espèces.

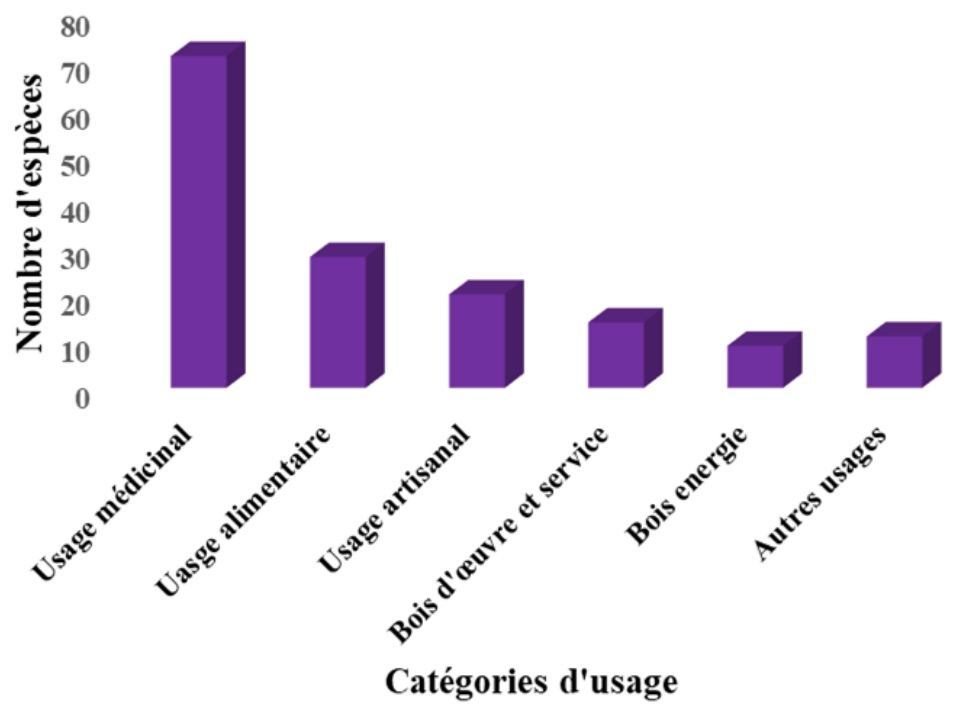

Figure 2. Répartition des espèces par catégories d'usage 


\section{Valeur d'importance des espèces utilitaires}

Les valeurs d'importance de 13 espèces citées par les populations varient de 50 à 66,67\% (Tableau 2). Ces espèces sont considérées comme les plus importantes pour les populations Elles sont utilisées au moins dans trois catégories d'usage. On a entre autres Ricinodendron heudelotii var. Africanum Baill. Pierre ex Heckel, Elaeis guineensis Jacq., Theobroma cacao Linn., Ceiba pentandra (L.) Gaertn., Spondias mombin L., Napoleonaea vogelii Hook. \& Planch. Nesogordonia papaverifera (A. Chev.) Capuron ex N. Hallé, Pycnanthus angolensis (Welw.) Warb., Carpolobia lutea G. Don, Celtis zenkeri Engl.

\section{Disponibilité des plantes utilitaires importantes dans la zone de conservation de biodiversité}

Parmi les espèces importantes, six (6) notamment Ricinodendron heudelotii var. Africanum Baill. Pierre ex Heckel, Carpolobia lutea G. Don sont moins abondantes dans la zone de conservation de biodiversité ; ce qui se traduit par leur indice de raréfaction supérieur à 80\% (Tableau 1). Les plus abondantes sont entre autres Elaeis guineensis Jacq., Theobroma cacao Linn., Ceiba pentandra (L.) Gaertn.

Tableau 1. Valeur d'importance et disponibilité de quelques espèces dans la zone de conservation de biodiversité

\begin{tabular}{|c|c|c|c|c|c|c|c|c|c|}
\hline $\mathrm{N}^{\circ}$ & Taxons & $\begin{array}{c}\text { Usage } \\
\text { alimentaire }\end{array}$ & $\begin{array}{c}\text { Usage } \\
\text { médicinal }\end{array}$ & $\begin{array}{c}\text { Usage } \\
\text { artisanal }\end{array}$ & BOS & $\begin{array}{l}\text { Bois } \\
\text { énergie }\end{array}$ & $\begin{array}{l}\text { Divers } \\
\text { usages }\end{array}$ & VI & IR \\
\hline 1 & $\begin{array}{l}\text { Ricinodendron } \\
\text { heudelotii var. } \\
\text { Africanum Baill. } \\
\text { Pierre ex Heckel }\end{array}$ & 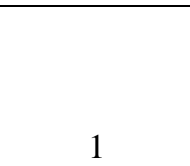 & 1 & 1 & & 1 & & 66,67 & 89,47 \\
\hline 2 & $\begin{array}{l}\text { Elaeis guineensis } \\
\text { Jacq. }\end{array}$ & 1 & 1 & 1 & & & & 50,00 & 26,32 \\
\hline 3 & $\begin{array}{c}\text { Theobroma cacao } \\
\text { Linn. }\end{array}$ & 1 & & & & 1 & 1 & 50,00 & 57,89 \\
\hline 4 & $\begin{array}{l}\text { Ceiba pentandra (L.) } \\
\text { Gaertn. }\end{array}$ & 1 & 1 & & 1 & & & 50,00 & 68,42 \\
\hline 5 & Spondias mombin L. & 1 & 1 & & & & 1 & 50,00 & 73,68 \\
\hline 6 & $\begin{array}{l}\text { Napoleonaea vogelii } \\
\text { Hook. \& Planch. }\end{array}$ & 1 & 1 & 1 & & & & 50,00 & 78,95 \\
\hline 7 & $\begin{array}{c}\text { Nesogordonia } \\
\text { papaverifera (A. } \\
\text { Chev.) Capuron ex N. } \\
\text { Hallé }\end{array}$ & & 1 & 1 & 1 & & & 50,00 & 78,95 \\
\hline 8 & $\begin{array}{c}\text { Pycnanthus } \\
\text { angolensis (Welw.) } \\
\text { Warb. } \\
\end{array}$ & & 1 & 1 & 1 & & & 50,00 & 78,95 \\
\hline 9 & $\begin{array}{l}\text { Carpolobia lutea } \\
\text { G.Don }\end{array}$ & 1 & 1 & 1 & & & & 50,00 & 84,21 \\
\hline 10 & Celtis zenkeri Engl. & & 1 & & 1 & 1 & & 50,00 & 84,21 \\
\hline
\end{tabular}




\begin{tabular}{c|c|c|c|c|c|c|c|c|c}
\hline $\mathbf{N}^{\mathbf{N}}$ & Taxons & $\begin{array}{c}\text { Usage } \\
\text { alimentaire }\end{array}$ & $\begin{array}{c}\text { Usage } \\
\text { médicinal }\end{array}$ & $\begin{array}{c}\text { Usage } \\
\text { artisanal }\end{array}$ & BOS & $\begin{array}{c}\text { Bois } \\
\text { énergie }\end{array}$ & $\begin{array}{c}\text { Divers } \\
\text { usages }\end{array}$ & VI & IR \\
\hline 11 & Carica papaya L. & 1 & 1 & & & & 1 & 50,00 & 89,47 \\
\hline & $\begin{array}{c}\text { Entandrophragma } \\
\text { angolense (Welw.) C. } \\
\text { DC. }\end{array}$ & 12 & 1 & 1 & 1 & & & & \\
\hline & $\begin{array}{c}\text { Bombax } \\
\text { buonopozense P. } \\
\text { Beauv. }\end{array}$ & 1 & 1 & & 1 & & & & \\
\hline
\end{tabular}

Légende : VI : Valeur d'importance ; IR : Indice de raréfaction ; BOS : Bois d'œuvre et service

\section{Restauration de la zone de conservation de biodiversité}

Le processus de reboisement a permis de repiquer en terre 17.566 plantules sur une superficie totale dégradée de 29 ha. La priorité a été portée dans un premier temps sur les espèces prioritaires pour la conservation selon l’UICN (2018) et Aké-Assi (1998) et dans un second temps sur les espèces utilitaires les plus importantes pour les populations riveraines.

\section{Discussion}

La formation et la sensibilisation des populations sur les biens et services écosystémiques a permis de faciliter l'acceptation sociale du projet. Ayant compris l'importance d'une aire protégée pour leur bien-être, les populations ont libéré les emprises du site afin que les activités d'aménagement soient mises en œuvre. Aussi, elles se sont-elles constituées d'une part en personnel d'appui pour aider les experts à bien accomplir leurs tâches et d'autre part en éco-garde pour la surveillance. Leur soutien a permis en premier lieu d'exécuter les tâches nécessitant de la main d'œuvre comme la collecte et production de plants en pépinière. En second lieu, leur appui a été nécessaire pour le planting de reboisement. Le choix des espèces à statut particulier et à haute valeur d'importance pour les populations pour le reboisement permettrait d'accroitre la valeur écologique du site au cours de sa reconstitution. L’appui des éco-gardes issus des communautés locales a été déjà expérimenté dans le cadre de la mise en place de la Réserve naturelle volontaire de la Dodo dans le département de Grand Béréby (Kouadio et al., 2018).

La flore initiale de la zone de conservation de biodiversité demeure riche avec 346 espèces reparties en 262 genres et 89 familles. Cette richesse floristique va dans le même ordre de grandeur que la richesse floristique de la zone de compensation écologique d'Agbaou Gold Operation dénommée réserve forestière Dekpa d'Agbaou (Vroh et al., 2014). Cette grande richesse observée peut se justifier par la diversité des biotopes inventoriés. En effet, l'on rencontre des espaces anciennement cultivés avec leur cortège d'espèces rudérales, des jachères riches en espèces pionnières, des zones marécageuses 
et des forêts galeries, qui abritent des espèces particulièrement adaptées à la vie au contact de l'eau. Aussi, la région dont est issue la zone de conservation de biodiversité et qui abrite le Parc National de Taï est connue comme le centre de gradients de diversités floristiques en Côte d'Ivoire (Kouamé et al., 2004).

La dominance des familles des Fabaceae, Rubiaceae, Euphorbiaceae est une caractéristique propre à l'ensemble des forêts ivoiriennes selon plusieurs travaux (Nusbaumer et al., 2005; N’Da et al., 2008; Kouamé et al., 2010; Vroh, 2013). Ces familles généralement de type Guinéo-Congolais et se développant dans les forêts denses humides (Kouamé, 2009), justifient d'une part la forte proportion des espèces qui se rencontrent en région GuinéoCongolaise (GC). D’autre part, la dominance des espèces de la région GuinéoCongolaise (GC) est une preuve que cette zone appartient bien à la région Guinéo Congolaise (Tiokeng et al., 2015). Cependant, leur proportion reste encore faible comparé au taux d'endémisme de $80 \%$ à $90 \%$ que la flore de cette région phytogéographique devrait avoir (Solefack et al., 2018). La zone de conservation de biodiversité constitue un refuge d'espèces prioritaires pour la conservation en ce sens qu'elle regorge une concentration d'espèces rares et/ou menacées d'extinction, ainsi que des espèces endémiques. En ce qui concerne les espèces rares et/ou menacées, 6 des 14 espèces (soit 42,86 \%) menacées de la flore ivoirienne ont été rencontrées sur le site de conservation. Ces résultats confirment l'importance de la zone pour la conservation de la flore endémique et menacée de cette région. Quant aux espèces endémiques, on y rencontre les 3 catégories signalées par Alonso et al. (2005) à savoir les espèces endémiques à l'Afrique de l'Ouest, les espèces endémiques ivoiriennes et les espèces " sassandriennes ». Ces dernières caractérisent la végétation comprise entre les fleuves Sassandra et Cavally. La présence de ces espèces endémiques témoigne d'un milieu relativement conservé (Adou Yao, 2005) et signe d'une grande biodiversité. Cette forte présence serait le fait de certains biotopes tels que les formations sur les îles qui ont échappées aux activités anthropiques du fait de leur accès difficile avant la construction du barrage. Aussi, l'on noterait-il la présence de quelques reliques de forêts secondaires épargnées par les populations qui auraient abrité ces espèces pendant la mise en place des plantations cacaoyères.

En ce qui concerne les espèces utilitaires, les populations en ont identifié plusieurs dans différents domaines. Certaines espèces sont utilisées à la fois dans plusieurs domaines. Ce qui fait d'elles des espèces à haute valeur d'importance pour les populations. La rencontre d'espèces à multiple usages dans une région révèle le savoir traditionnel élevé des populations (Makumbelo et al., 2008). Cette caractéristique dévolue à ces plantes repose sur leurs organisations biologiques complexes (Ozenda, 2007). En effet, elles produisent divers organes dont chacun pourrait être utilisé en réponse à un besoin spécifique des communautés rurales. Ces espèces végétales constituent 
des moyens de subsistance eu égard aux valeurs sociales et économiques attachées à chacune d'elle (Neffati et al., 2016).

La rareté de certaines plantes utilitaires importantes dans la zone de conservation de biodiversité serait due à leur grande sollicitation et leur mode de prélèvement. Ce dernier se fait dans la plupart des cas par écorçage et coupe de racines et tiges. Ces modes de prélèvement couplés à l'intensité de prélèvement leur rendraient hautement vulnérable (Makumbelo et al., 2008). En effet, il existe d'une part, une relation manifeste entre la partie de la plante exploitée et la régénération des espèces et d'autre part, entre le mode de prélèvement et l'intensité de prélèvement sur la régénération des espèces (Dossou et al., 2012). Ainsi, il est important de sensibiliser les populations sur les techniques rationnelles de prélèvement des organes des plantes afin de ne pas entamer la possibilité de bénéficier durablement des services de ces plantes à haute valeur d'importance.

Des auteurs reconnaissent que la valeur d'importance d'une plante peut expliquer sa préservation et l'attribution d'une place spéciale dans le système de gestion du terroir (Guillemette, 2014). Ainsi, pour éviter la perte de ces ressources, ces espèces ont constitué une priorité dans le choix des espèces de reboisement afin d'enrichir la zone de conservation de biodiversité en espèces utilitaires de première nécessité pour les populations.

\section{Conclusion}

L'étude menée dans la zone de conservation de biodiversité du barrage hydroélectrique de Soubré a permis de mettre en évidence l'impact du séminaire de formation et de sensibilisation des populations sur l'importance de la gestion et de la conservation de la biodiversité du site vue les biens et les services rendus par les écosystémiques de manière générale. Elle aurait facilité l'acceptation sociale du projet de gestion durable des ressources naturelles. Aussi, l'implication des communautés locales aux différentes étapes du processus a-t-elle été d'un atout important. Le bon déroulement des travaux a permis de restaurer les sites dégradés de la zone avec 17.566 plantules pour une superficie totale dégradée de 29 ha. Le but étant d'améliorer la valeur écologique de la zone de conservation de biodiversité qui regorge plusieurs biotopes riches de 346 espèces végétales reparties en 262 genres et 89 familles. Au sein de ces espèces, 50 détiennent un statut de conservation. En ce qui concerne les espèces utilitaires de la région, 13 sont plus importantes. Cependant, six d'entre elles sont moins abondantes dans la zone de conservation de biodiversité. Ce sont ces dernières et celles à statut de conservation qui ont été priorisées au cours de la restauration. 


\section{Annexe}

Annexe 1. Liste des espèces à statut particulier

\begin{tabular}{|c|c|c|c|c|c|}
\hline $\mathbf{N}^{\mathbf{0}}$ & Espèces & Familles & Endémisme & $\begin{array}{l}\text { Statut } \\
\text { UICN } \\
(2018)\end{array}$ & $\begin{array}{l}\text { Statut Aké- } \\
\text { Assi (1998) }\end{array}$ \\
\hline 1 & Afzelia bella var. gracilior Keay & Fabaceae & GCW & & \\
\hline 2 & Albizia ferruginea (Guill. \& Perr.) Benth. & Fabaceae & & VU & \\
\hline 3 & Ancistrocladus barteri Sc. Elliot & Ancistrocladaceae & GCW/HG & & \\
\hline 4 & Baissea zygodioides (K. Schum.) Stapf & Apocynaceae & HG & & \\
\hline 5 & Baphia pubescens Hook. f. & Fabaceae & GCi & & \\
\hline 6 & $\begin{array}{c}\text { Brachystegia leonensis Burtt Davy \& } \\
\text { Hutch. }\end{array}$ & Fabaceae & GCW/HG & & \\
\hline 7 & Bussea occidentalis Hutch. & Fabaceae & HG & & \\
\hline 8 & Chassalia afzelii (Hiern) K. Schum. & Rubiaceae & GCW & & \\
\hline 9 & Cissus miegei Tchoumé & Vitaceae & GCi/HG & & \\
\hline 10 & Cola caricifolia (G. Don) K. Schum. & Malvaceae & GCW/HG & & \\
\hline 11 & Combretum comosum G. Don & Combretaceae & GCW & & \\
\hline 12 & Copaifera salikounda Heckel & Fabaceae & GCW/HG & VU & \\
\hline 13 & Craibia atlantica Dunn & Fabaceae & & VU & \\
\hline 14 & Croton scarciesii Scott-Elliot & Euphorbiaceae & GCW & & \\
\hline 15 & Dialium aubrevillei Pellegr. & Fabaceae & GCW/HG & & \\
\hline 16 & Dichapetalum toxicarium (G. Don) Baill. & Dichapetalaceae & HG & & \\
\hline 17 & Dictyophleba leonensis (Stapf) Pichon & Apocynaceae & GCW & & \\
\hline 18 & Diospyros heudelotii Hiern & Ebenaceae & GCW/HG & & \\
\hline 19 & $\begin{array}{c}\text { Entandrophragma angolense (Welw.) C. } \\
\text { DC. }\end{array}$ & Meliaceae & & VU & \\
\hline 20 & $\begin{array}{l}\text { Entandrophragma cylindricum (Sprague) } \\
\text { Srague }\end{array}$ & Meliaceae & & VU & \\
\hline 21 & Euadenia eminens Hook. f & Capparaceae & GCW/HG & & \\
\hline 22 & Eugenia gabonensis Amshoff & Myrtaceae & GCi & & \\
\hline 23 & Garcinia afzelii Engl. & Clusiaceae & & VU & PRE \\
\hline 24 & $\begin{array}{c}\text { Hibiscus comoensis A. Chev. ex Hutch. \& } \\
\text { Dalziel }\end{array}$ & Malvaceae & GCi/HG & & PRE \\
\hline 25 & $\begin{array}{c}\text { Isolona cooperi Cooper \& Record ex Hutch. } \\
\text { \& Dalziel }\end{array}$ & Annonaceae & GCW/HG & & \\
\hline 26 & $\begin{array}{c}\text { Lannea nigritana (Sc. Elliot) Keay var. } \\
\text { pubescens Keay }\end{array}$ & Anacardiaceae & & & PRE \\
\hline 27 & Leptoderris miegei Aké Assi \& Mangenot & Fabaceae & GCi/HG & & \\
\hline 28 & Maranthes robusta (Oliv.) France & Chrysobalanaceae & & & PRE \\
\hline 29 & Milicia excelsa (Welw.) C.C. Berg & Moraceae & & & PRE \\
\hline
\end{tabular}




\begin{tabular}{|c|c|c|c|c|c|}
\hline $\mathbf{N}^{\mathbf{0}}$ & Espèces & Familles & Endémisme & (2018) & Assi (1998) \\
\hline 30 & Milicia regia (A. Chev.) C.C. Berg & Moraceae & GCW/HG & VU & PRE \\
\hline 31 & Millettia lane-poolei Dunn & Fabaceae & GCW/HG & & \\
\hline 32 & Millettia lucens (Scott- Elliot) Dunn & Fabaceae & GCW/HG & & \\
\hline 33 & $\begin{array}{l}\text { Mitragyna ledermannii (K. Krause) } \\
\text { Ridsdale }\end{array}$ & Rubiaceae & & VU & \\
\hline 34 & Mussaenda chippii Wernham & Rubiaceae & GCW/HG & & \\
\hline 35 & Myrianthus libericus Rendle & Urticaceae & HG & & \\
\hline 36 & $\begin{array}{l}\text { Nesogordonia papaverifera (A. Chev.) } \\
\text { Capuron ex N. Hallé }\end{array}$ & Malvaceae & & VU & \\
\hline 37 & Neuropeltis prevosteoides Mangenot & Convolvulaceae & GCW/HG & & \\
\hline 38 & Pararistolochia mannii (Hook.f.) Keay & Aristolochiaceae & & & PRE \\
\hline 39 & Penianthus zenkeri (Engl.) Diels & Mennispermaceae & GCW & & \\
\hline 40 & Platysepalum hirsutum (Dunn) Hepper & Fabaceae & GCW/HG & & \\
\hline 41 & Rinorea subintegrifolia (P. Beauv.) Kuntze. & Violaceae & GCW & & \\
\hline 42 & Salacia lateritia N. Hallé & Celastraceae & HG & & \\
\hline 43 & Sherbournia calycina (G.Don) Hua & Rubiaceae & GCW/HG & & \\
\hline 44 & Strychnos dinklagei Gilg & Loganiaceae & GCW/HG & & \\
\hline 45 & Tiliacora leonensis (Scott-Elliot) Diels & Mennispermaceae & GCW & & \\
\hline 46 & Triclisia patens Oliv. & Mennispermaceae & GCW/HG & & \\
\hline 47 & Urera keayi Letouzey & Urticaceae & HG & & \\
\hline 48 & Uvaria ovata subsp. afzeliana (DC.) Keay & Annonaceae & & & PRE \\
\hline 49 & Uvariodendron occidentale Le Thomas & Annonaceae & GCW/HG & VU & \\
\hline 50 & Xylia evansii Hutch. & Fabaceae & GCW/HG & & \\
\hline
\end{tabular}

Légende: GCi : taxon endémique à la Côte d'Ivoire ; GCW : taxon endémique du bloc forestier à l'ouest du Togo, comprenant le Ghana, la Côte d'ivoire, le Libéria, la Sierra Léone, la Guinée, la Guinée Bissau, la Gambie et le Sénégal ; HG : taxon de la haute guinée ; PRE : plantes rares, devenues rares et en voie d'extinction ; VU : vulnérable.

\section{References :}

1. Adou Yao, C. Y. (2005). Pratiques paysannes et dynamiques de la biodiversité dans la forêt classée de Monogaga (Côte d’Ivoire), Thèse de Doctorat unique, département Hommes Natures Sociétés, MNHN, Paris, France. 248p.

2. Aké-Assi, L. (1984). Flore de la Côte d'Ivoire : Etude descriptive et biogéographique avec quelques notes ethnobotaniques. Thèse de Doctorat d'Etat, Faculté des Sciences et Techniques, Université de Cocody, Abidjan (Côte d’Ivoire). 1206p. 
3. Aké-Assi, L. (1998). Impact de l'exploitation forestière et du développement agricole sur la conservation de la biodiversité biologique en Côte d'Ivoire. Le flambloyant 46 : 20 - 21 .

4. Aké-Assi, L. (2001). Flore de Côte d'Ivoire 1, catalogue systématique, biogéographie et écologie. Conservatoire et Jardin Botaniques, Genève, Suisse. 396pp.

5. Aké-Assi, L. (2002). Flore de la Côte d’Ivoire 2, catalogue systématique, biogéographie et écologie. Conservatoire et Jardin Botanique, Genève, Suisse. 441p.

6. Alonso, L. E., Lauginie, F., \& Rondeau, G. (2005). Une évaluation biologique de deux forêts classées du sud-ouest de la Côte d'Ivoire (pp. 91 - 100), Conservation International, Washington D.C, USA.

7. APG IV (2016). An update of the Angiosperm Phylogeny Group classification for the ordersand families of flowering plants. Botanical Journal of the Linnean Society 181: 1-20.

8. Dossou, M. E., Houessou, G. L., Lougbégnon, O. T., Tenté, A. H. B., \& Codjia, J. T. C. (2012). Ethnobotanical study of ligneous species in Agonvè swampy forest and surrounding area in Benin. Tropicultura $30(1): 41$ - 48.

9. FAO-REDD+ (2016). Données forestières de base pour la REDD+ en en Côte d'Ivoire : cartographie de la dynamique forestière de 1986 à 2015, rapport d'étude. 32pp.

10. Géhu, J. M., \& Géhu, J. (1980). Essai d'objection de l'évaluation biologique des milieux naturels. Exemples littoraux. In Géhu J.M. (Eds.). Séminaire de Phytosociologie Appliquée (pp. 75-94), Amicale Francophone de Phytociologie, Metz, France.

11. Guillemette, E. (2014). Caractérisation et suivi des attributs multiressources de la Forêt habitée du Massif dans un contexte d'aménagement durable. Rapport de Maîtrise en science forestière, Université de Laval, Québec, Canada. 65 pp.

12. Konan, K. E. (2008). Conservation de la diversité végétale et activités humaines dans les aires protégées du sud forestier ivoirien : l'exemple du Parc National d'Azagny. Thèse Unique Université d'AbidjanCocody, Côte d'Ivoire. 210pp.

13. Kouadio, Y. J-C., Soiret, K. S. P., Kpan, W. B., Yao, N. O., N'guessan, K. E., Kouassi, K. P., \& Gomez, P. J. (2018). Valeur de conservation de la Réserve Naturelle Volontaire (RNV) de la Dodo, Sud-Ouest de la Côte d'Ivoire (Afrique de l'Ouest), International Journal of Biological and Chemical Sciences 12(6) : 2784-2796 
14. Kouamé, D., Adou Yao, C. Y., Nandjui, A., \& N'Guessan, E. K. (2010). Le rôle de l'éléphant dans la germination des graines de Irvingia gabonensis (Irvingiaceae), Balanites wilsoniana (Balanitaceae), Parinari excelsa (Chrysobalanaceae) et Sacoglottis gabonensis (Humiriaceae) en forêt tropicale : cas du Parc National d'Azagni. International Journal of Biological and Chemical Sciences $4(5): 1442-1454$.

15. Kouamé, D. (2009). Rôle des animaux frugivores dans la régénération et la conservation des forêts : cas de l'éléphant (Loxodonta africanacyclotis Matschié, 1900) dans le Parc National d'Azagny (Sud-est de la Côte d'Ivoire). Thèse de Doctorat, Université de Cocody-Abidjan, Côte d'Ivoire. 215pp.

16. Kouamé, N. F., Kouadio, K. E., Kouassi, K., \& Poorter, L. (2004). Floristic diversity of closed forests in Côte d'Ivoire. In Poorter L, Bongers F, Kouame FN. \& Hawthorne W. (Eds.). Biodiversity of West African forests: an ecological atlas of woody plant species (pp. 53-60), Colophon, Wageningen.

17. Lanly, J. P. (1969). Régression de la forêt dense en Côte-d'Ivoire. Bois et forets des tropiques 127(127) : 45 - 59.

18. Lebrun, J. P., \& Stork, A. L. (1991-2015). Enumération des plantes à fleurs d'Afrique tropicale. Conservatoire et Jardin Botaniques de la ville de Genève, 4 volumes. https://www.villege.ch/musinfo/bd/cjb/africa/recherche.php?langue $=\mathrm{fr}$

19. Makumbelo, E., Lukoki, L., Paulus, J. J. S. J., \& Luyindula, N. (2008). Stratégie de valorisation des espèces ressources des produits non ligneux de la savane des environs de Kinshasa : II. Enquête ethnobotanique (aspects médicinaux). Tropicultura 26(3) : 129 - 134.

20. MINEF (2020). Stratégie nationale de préservation, de réhabilitation et d'extension des forêts, 52p

21. N’Da, D., Adou Yao, C. Y., N'guessan, K. E., Kone, M., \& Sagne, Y. C. (2008). Analyse de la diversité floristique du parc national de la Marahoué, Centre-Ouest de la Côte d'Ivoire. Afrique Science 4(3) : $552-579$.

22. Neffati, M., Sghaier, M., \& Labbene, Y. (2016). Analyse de la vulnérabilité des écosystèmes et des moyens de subsistance Livelihoods) des populations au changement climatique en zones arides et désertiques de la région MENA. Rapport principal, Centre national de documentation, Rabat, Maroc. 202 pp

23. Nusbaumer, L., Gautier, L., Chatelain, C., \& Spichiger, R. (2005). Structure et composition floristique de la Forêt Classée du Scio (Côte d'Ivoire), Etude descriptive et comparative. Candollea 60 (2) : 393 443. 
24. Ozenda, P. (2007). Les végétaux : organisation et diversité biologique. Ed. Dunod, Grenoble, France. 528pp.

25. Poorter, L., Bongers, F., Kouamé, F. N., \& Hawthorne, W. D. (2004). Biodiversity of West African Forests: An Ecological Atlas of Woody Plant Species. CABI Publishing, Netherland, Pays-Bas, 521pp.

26. Solefack, M. C. M., Temgoua, L. F., Fedoung, E., \& Djouba, R. Z. (2018). Végétation et spectres fonctionnels de la galerie forestière de Koupa Matapit (Ouest-Cameroun). GeoEco-Trop 42 (1) : 147 - 158.

27. Tiokeng, B., Mapongmetsem, P., Nguetsop, V. F., \& Tacham, W. N. (2015). Biodiversité floristique et régénération naturelle sur les Hautes Terre de Lebialem (Ouest Cameroun). International Journal of Biological and Chemical Sciences 9(1) : 56 - 68.

28. UICN (2018). IUCN Red List of Threatened Species. [Online] Available: http://www.iucnredlist.org (Avril 2018)

29. Vroh, B. T. A. (2013). Evaluation de la dynamique de la végétation dans les zones agricoles d'Azaguié (Sud-Est, Côte d'Ivoire). Thèse de Doctorat, Université Félix Houphouët Boigny, Abidjan, Côte d'Ivoire. 208pp.

30. Vroh B. T. A., Tiébré, M-S., Ouattara, D., \& N'Guessan, K. E. (2014). La réserve forestière Dékpa d'Agbaou, un exemple de conservation de la diversité végétale sur les sites miniers de la Côte d'Ivoire, International Journal of Innovation and Applied Studies 6(2) : 162-171 